\title{
Crystal Structure of Ruthenium Phthalocyanine with Diaxial Monoatomic Ligand: Bis(Triphenylphosphine)Iminium Dichloro(Phthalocyaninato(2-))Ruthenium(III)
}

\author{
Derrick Ethelbhert Yu, ${ }^{1}$ Akira Kikuchi, ${ }^{2}$ Tetsuya Taketsugu, ${ }^{2}$ and Tamotsu Inabe ${ }^{2}$ \\ ${ }^{1}$ Department of Chemistry, College of Science, De La Salle University, 2401 Taft Avenue, Manila 1004, Philippines \\ ${ }^{2}$ Division of Chemistry, Graduate School of Science, Hokkaido University, Sapporo 060-0810, Japan \\ Correspondence should be addressed to Derrick Ethelbhert Yu; derrick.yu@dlsu.edu.ph
}

Received 27 June 2012; Accepted 6 September 2012

Academic Editor: Mitsushiro Nomura

Copyright (C) 2013 Derrick Ethelbhert Yu et al. This is an open access article distributed under the Creative Commons Attribution License, which permits unrestricted use, distribution, and reproduction in any medium, provided the original work is properly cited.

\begin{abstract}
Axially-ligated iron phthalocyanines have been found to be good molecular conductors with giant negative magnetoresistance (GNMR) which originates from a strong intramolecular $\pi$ - $d$ interaction between the metal and phthalocyanine. Ab initio theoretical calculations showed that substitution of ruthenium into the phthalocyanine complex would result in a significant increase in the $\pi-d$ interaction of the system, potentially intensifying GNMR. This paper presents the crystal preparation and X-ray structural characterization of bis(triphenylphosphine)iminium dichloro(phthalocyaninato(2-))ruthenium(III), $\mathrm{PNP}\left[\mathrm{Ru}^{\mathrm{III}}\left(\mathrm{Pc}^{2-}\right) \mathrm{Cl}_{2}\right]$. It is observed that $\left[\mathrm{Ru}^{\mathrm{III}}\left(\mathrm{Pc}^{2-}\right) \mathrm{Cl}_{2}\right]$ system has a symmetric planar $\mathrm{RuPc}$ unit with perpendicular axial ligands which results in a unidirectional and uniform solid-state arrangement, suitable for $\pi-d$ interaction-based molecular conductors with potentially exceptional GNMR.
\end{abstract}

\section{Introduction}

Metallophthalocyanine complexes with mono- or diatomic linear diaxial ligands (Scheme 1) are suitable molecular conductors due to their ability to form a slip-stacked solidstate arrangement that permits intermolecular $\pi-\pi$ overlap for electron conduction [1, 2]. Moreover, the existence of strong intramolecular $\pi$ - $d$ interaction in axially ligated iron(III) phthalocyanine $\left(\mathrm{Fe}^{\mathrm{III}}(\mathrm{Pc}) \mathrm{L}_{2}\right.$; where $\mathrm{L}=\mathrm{CN}, \mathrm{Cl}$, $\mathrm{Br})$ molecular conductors has resulted in anisotropic giant negative magnetoresistance (GNMR) of up to $95 \%$ decrease in electrical resistance at 15 Tesla [3].

$\mathrm{Ab}$ initio theoretical calculations using MOLPRO software package [4] performed on the $\mathrm{D}_{4 h} \mathrm{Fe}^{\mathrm{III}}(\mathrm{Pc}) \mathrm{L}_{2}$ system corroborated experimental observation that the strength of GNMR is directly related to the strength of $\pi$ - $d$ interaction in the order of $\mathrm{L}=\mathrm{CN}>\mathrm{Cl}>\mathrm{Br}$. On the electronic structure representation of $\left[\mathrm{Fe}^{\mathrm{III}}(\mathrm{Pc}) \mathrm{L}_{2}\right]$ species, the $\mathrm{Fe}^{3+} d^{5}$ configuration gives two-fold degenerate $\left(d_{x y}\right)^{2}\left(d_{x z}\right)^{2}\left(d_{y z}\right)^{1}$ $=\left(d_{x y}\right)^{2}\left(d_{x z}\right)^{1}\left(d_{y z}\right)^{2}$ while the HOMO is a singly occupied molecular orbital of the delocalized $\pi$-system of the Pc. Electronic structure calculations using two state-averaged complete active space multiconfigurational SCF method (active space orbitals: Pc- $\pi$, Fe- $d_{x z}$ and $d_{y z}$; Stuttgart-Köln $\mathrm{ECP}+\mathrm{DZ}$ basis) resulted in $\Delta E$ (orbital energy difference between $d_{y z} / d_{x z}$ and HOMO; intensity of the $\pi-d$ interaction) of $8.5450 \mathrm{eV}, 8.3839 \mathrm{eV}$, and $7.8655 \mathrm{eV}$ for $\mathrm{L}=$ $\mathrm{Br}, \mathrm{Cl}$, and $\mathrm{CN}$, respectively. Using the same theoretical calculation framework to $\mathrm{Ru}^{\mathrm{III}}(\mathrm{Pc}) \mathrm{L}_{2}$, which is electronically isostructural with the $\mathrm{Fe}{ }^{\mathrm{III}}(\mathrm{Pc}) \mathrm{L}_{2}$ species, the $d^{5}$ homologue system resulted in a remarkable increase of around two-fold in the $\pi$ - $d$ interactions across all $\mathrm{Ru}^{\mathrm{III}}(\mathrm{Pc}) \mathrm{L}_{2}$ species (L: $\mathrm{CN}=3.7518 \mathrm{eV}, \mathrm{Cl}=3.8419 \mathrm{eV}, \mathrm{Br}=3.9411 \mathrm{eV})$. Given that the intensity of the unique intramolecular $\pi$ - $d$ interaction as the origin of the varying anisotropic GNMR in $\mathrm{M}^{\mathrm{III}}(\mathrm{Pc}) \mathrm{L}_{2}$, thus the importance of the synthesis of ruthenium(III) phthalocyanine with linear axial ligands.

The synthesis of crystalline ruthenium phthalocyanine $\mathrm{Ru}(\mathrm{Pc})$ complexes has long been a challenge in phthalocyanine chemistry. Even upon the report of pure $\mathrm{Ru}(\mathrm{Pc})$ 
TABLE 1: Crystallographic data collection parameters of $\mathrm{PNP}\left[\mathrm{Ru}^{\mathrm{III}}(\mathrm{Pc}) \mathrm{Cl}_{2}\right]$ at $123 \mathrm{~K}$.

\begin{tabular}{ll}
\hline Empirical formula & $\mathrm{C}_{68} \mathrm{H}_{46} \mathrm{~N}_{9} \mathrm{Cl}_{2} \mathrm{P}_{2} \mathrm{Ru}_{1}$ \\
Formula weight & 1223.10 \\
Crystal system & Triclinic \\
& $a=10.4425(11) \AA$ \\
& $b=12.2391(11) \AA$ \\
& $c=13.159(11) \AA$ \\
Lattice parameters & $\alpha=75.523(3)^{\circ}$ \\
& $\beta=64.686(3)^{\circ}$ \\
& $\gamma=65.883(3)^{\circ}$ \\
& $V=1381.9(2) \AA^{3}$ \\
Space group & $P \overline{1}(\# 2)$ \\
$Z$ value & 1 \\
Calculated density & $1.470 \mathrm{~g} / \mathrm{cm}^{3}$ \\
$\mu($ MoK $\alpha)$ & $4.92 \mathrm{~cm}^{-1}$ \\
$2 \theta_{\text {max }}$ & $49.0^{\circ}$ \\
Reflections collected/unique & $10394 / 4588$ \\
$R 1[I>2.00 \sigma(I)]$ & {$[R(\mathrm{int})=0.1173]$} \\
$w R 2($ all data $)$ & 0.0803 \\
Goodness-of-fit indicator & 0.2345 \\
\hline
\end{tabular}

synthesis more than three decades ago, the ambiguities of its solid-state/materials science still remain as only very few crystal structures of 6-coordinated axially ligated $\mathrm{Ru}(\mathrm{Pc})$ complexes have been reported $[5,6]$. However, these $\mathrm{Ru}(\mathrm{Pc})$ complexes have bulky and/or unsymmetrical axial ligands unsuitable for structure-property correlation studies. To date, only one axially-ligated magnetic $\mathrm{Ru}^{3+}\left(d^{5}\right)$-centered Pc crystal has been reported. Yet, this reported $\mathrm{Ru}^{\mathrm{III}}(\mathrm{Pc}) \mathrm{L}_{2}$ crystal has unsymmetrical mixed axial cyano and pyridine ligands from an attempted identical di-axial ligation synthesis [7]. Herein, we report the crystal structure of ruthenium(III) phthalocyanine with identical di-axial linear ligands which can form symmetrical octahedral architecture that could be a potential component for magnetotransport material application.

\section{Methodology}

2.1. Crystallization. Dichloro(phthalocyaninato(1-)) ruthenium(III), $\mathrm{Ru}^{\mathrm{III}}\left(\mathrm{Pc}^{1-}\right) \mathrm{Cl}_{2}$, was prepared via the method reported by Myers et al. in preparing various $\mathrm{M}^{\mathrm{III}}\left(\mathrm{Pc}^{1-}\right) \mathrm{Cl}_{2}$ through the reaction of $\mathrm{M}^{\mathrm{II}}(\mathrm{Pc})$ with thionyl chloride oxidizing agent [8]. Ru ${ }^{\mathrm{II}} \mathrm{Pc}$ (500 mg; $0.81 \mathrm{mmol}$ ) synthesized using the procedure of Farrell et al. [9], was suspended in nitrobenzene $(10 \mathrm{~mL})$. Thionyl chloride $(2 \mathrm{~mL} ; 28 \mathrm{mmol})$ was subsequently added to the reaction vessel and refluxed at $70^{\circ} \mathrm{C}$ for 3 hours. A 1:10 mole ratio of $\mathrm{Ru}^{\mathrm{III}}\left(\mathrm{Pc}^{1-}\right) \mathrm{Cl}_{2}$ and bis(triphenylphosphine) iminium chloride (PNPCl) was dissolved in a $1: 1: 1: 1$ (volume) dimethylformamide:acetone: ethanol: hexane solvent system. The resulting solution
TABLE 2: Intramolecular bond lengths ( $\AA$ ) of $\mathrm{PNP}\left[\mathrm{Ru}^{\mathrm{III}}(\mathrm{Pc}) \mathrm{Cl}_{2}\right]$.

\begin{tabular}{|c|c|}
\hline Atom & Distance \\
\hline Ru1-N3 & $1.982(9)$ \\
\hline Ru1-N1 & $1.993(8)$ \\
\hline P1-N5 & $1.552(3)$ \\
\hline $\mathrm{P} 1-\mathrm{C} 23$ & $1.796(10)$ \\
\hline N2-C8 & $1.335(12)$ \\
\hline N3-C16 & $1.395(12)$ \\
\hline N5-P1 & $1.552(3)$ \\
\hline C2-C3 & $1.380(13)$ \\
\hline $\mathrm{C} 3-\mathrm{H} 3$ & 0.9300 \\
\hline C5-C6 & $1.396(14)$ \\
\hline C6-H6 & 0.9300 \\
\hline C10-C11 & $1.383(14)$ \\
\hline C11-H11 & 0.9300 \\
\hline C13-C14 & $1.384(15)$ \\
\hline C14-H14 & 0.9300 \\
\hline C17-C18 & $1.402(16)$ \\
\hline C19-C20 & $1.353(19)$ \\
\hline C20-H20 & 0.9300 \\
\hline $\mathrm{C} 22-\mathrm{H} 22$ & 0.9300 \\
\hline C24-C25 & $1.371(15)$ \\
\hline C25-H25 & 0.9300 \\
\hline C27-C28 & $1.359(15)$ \\
\hline C29-C30 & $1.375(13)$ \\
\hline С30-H30 & 0.9300 \\
\hline C32-C33 & $1.354(14)$ \\
\hline C33-H33 & 0.9300 \\
\hline Ru1-N3 & $1.982(9)$ \\
\hline Ru1-Cl1 & $2.355(3)$ \\
\hline $\mathrm{P} 1-\mathrm{C} 17$ & $1.767(12)$ \\
\hline N1-C1 & $1.363(12)$ \\
\hline $\mathrm{N} 2-\mathrm{C} 9$ & $1.349(12)$ \\
\hline $\mathrm{N} 4-\mathrm{C} 1$ & $1.329(12)$ \\
\hline $\mathrm{C} 1-\mathrm{N} 4$ & $1.329(12)$ \\
\hline $\mathrm{C} 2-\mathrm{C} 7$ & $1.424(13)$ \\
\hline C4-C5 & $1.373(15)$ \\
\hline C5-H5 & 0.9300 \\
\hline C7-C8 & $1.463(13)$ \\
\hline C10-C15 & $1.417(14)$ \\
\hline $\mathrm{C} 12-\mathrm{C} 13$ & $1.406(15)$ \\
\hline C13-H13 & 0.9300 \\
\hline C15-C16 & $1.449(14)$ \\
\hline C18-C19 & $1.421(18)$ \\
\hline C19-H19 & 0.9300 \\
\hline C21-C22 & $1.389(18)$ \\
\hline C23-C28 & $1.382(13)$ \\
\hline C24-H24 & 0.9300 \\
\hline C26-C27 & $1.391(16)$ \\
\hline C27-H27 & 0.9300 \\
\hline C29-C34 & $1.403(14)$ \\
\hline C31-C32 & $1.390(16)$ \\
\hline C32-H32 & 0.9300 \\
\hline
\end{tabular}


TABLE 2: Continued.

\begin{tabular}{|c|c|}
\hline Atom & Distance \\
\hline C34-H34 & 0.9300 \\
\hline Ru1-N1 & $1.993(8)$ \\
\hline Ru1-Cl1 & $2.355(3)$ \\
\hline P1-C29 & $1.789(10)$ \\
\hline N1-C8 & $1.392(12)$ \\
\hline N3-C9 & $1.365(13)$ \\
\hline N4-C16 & $1.337(12)$ \\
\hline $\mathrm{C} 1-\mathrm{C} 2$ & $1.463(13)$ \\
\hline $\mathrm{C} 3-\mathrm{C} 4$ & $1.379(14)$ \\
\hline C4-H4 & 0.9300 \\
\hline C6-C7 & $1.373(13)$ \\
\hline C9-C10 & $1.481(13)$ \\
\hline $\mathrm{C} 11-\mathrm{C} 12$ & $1.376(14)$ \\
\hline C12-H12 & 0.9300 \\
\hline C14-C15 & $1.362(13)$ \\
\hline $\mathrm{C} 17-\mathrm{C} 22$ & $1.384(16)$ \\
\hline C18-H18 & 0.9300 \\
\hline C20-C21 & $1.34(2)$ \\
\hline $\mathrm{C} 21-\mathrm{H} 21$ & 0.9300 \\
\hline C23-C24 & $1.386(14)$ \\
\hline C25-C26 & $1.404(16)$ \\
\hline C26-H26 & 0.9300 \\
\hline C28-H28 & 0.9300 \\
\hline C30-C31 & $1.398(15)$ \\
\hline C31-H31 & 0.9300 \\
\hline C33-C34 & $1.361(15)$ \\
\hline
\end{tabular}

was then left in an evacuated dessicator compartment at $25^{\circ} \mathrm{C}$. Bis(triphenylphosphine)iminium dichloro(phthalocyaninato(2-)) ruthenium(III), PNP $\left[\mathrm{Ru}^{\mathrm{III}}\left(\mathrm{Pc}^{2-}\right) \mathrm{Cl}_{2}\right]$, crystallized into dark blue crystals after 8 weeks.

2.2. X-Ray Crystal Structure Determination. A blue block crystal of PNP $\left[\mathrm{Ru}^{\mathrm{III}}\left(\mathrm{Pc}^{2-}\right) \mathrm{Cl}_{2}\right.$ ] (Formula: $\mathrm{C}_{68} \mathrm{H}_{46} \mathrm{~N}_{9} \mathrm{Cl}_{2}$ $\mathrm{RuP}_{2}$ ) having approximate dimensions of $0.15 \times 0.10 \times$ $0.05 \mathrm{~mm}$ was mounted on a glass fiber. All measurements were made on a Rigaku RAXIS RAPID imaging plate area detector with graphite monochromated Mo-K $\alpha$ radiation. Indexing was performed from 3 oscillations that were exposed for 90 seconds. The crystal-to-detector distance was $127.40 \mathrm{~mm}$. The data were collected at a temperature of $123 \mathrm{~K}$ to a maximum $2 \theta$ value of $49.0^{\circ}$. A total of 44 oscillation images were collected. A sweep of data was done using $\omega$ scans from 130.0 to $190.0^{\circ}$ in $5.0^{\circ}$ step, at $\chi=45.0^{\circ}$ and $\phi=0.0^{\circ}$. The exposure rate was $150.0\left[\mathrm{sec} /^{\circ}\right]$. A second sweep was performed using $\omega$ scans from 0.0 to $160.0^{\circ}$ in $5.0^{\circ}$ step, at $\chi=45.0^{\circ}$ and $\phi=180.0^{\circ}$. The exposure rate was $150.0\left[\mathrm{sec} /^{\circ}\right]$. The crystal-to-detector distance was $127.40 \mathrm{~mm}$. Readout was performed in the $0.100 \mathrm{~mm}$ pixel mode. All post measurement data processing was performed using the CrystalStructure crystallographic software package [10].
TABLE 3: Intramolecular bond angles $\left({ }^{\circ}\right)$ of $\mathrm{PNP}\left[\mathrm{Ru}^{\mathrm{III}}(\mathrm{Pc}) \mathrm{Cl}_{2}\right]$.

\begin{tabular}{|c|c|}
\hline Atom & Angle \\
\hline N3 Ru1 N3 & $179.999(1)$ \\
\hline N3 Ru1 N1 & $90.3(3)$ \\
\hline N3 Ru1 Cl1 & $90.4(2)$ \\
\hline N1 Ru1 Cl1 & $91.6(2)$ \\
\hline N1 Ru1 Cl1 & $91.6(2)$ \\
\hline N5 P1 C17 & $110.3(4)$ \\
\hline N5 P1 C23 & $111.2(3)$ \\
\hline C1 N1 C8 & $109.8(8)$ \\
\hline C8 N2 C9 & $122.7(8)$ \\
\hline C16 N3 Ru1 & $125.7(6)$ \\
\hline N4 C1 N1 & $128.8(9)$ \\
\hline C3 C2 C7 & $120.0(9)$ \\
\hline C4 C3 C2 & $117.9(10)$ \\
\hline C5 C4 C3 & $122.2(10)$ \\
\hline C4 C5 C6 & $121.2(10)$ \\
\hline C7 C6 C5 & $117.3(10)$ \\
\hline C6 C7 C2 & $121.4(9)$ \\
\hline N2 C8 N1 & 127.7(9) \\
\hline N2 C9 N3 & 128.3(9) \\
\hline C11 C10 C15 & $120.9(9)$ \\
\hline C12 C11 C10 & $117.3(10)$ \\
\hline C11 C12 C13 & $122.0(10)$ \\
\hline C14 C13 C12 & $120.2(10)$ \\
\hline C15 C14 C13 & $118.5(10)$ \\
\hline C14 C15 C10 & $121.1(10)$ \\
\hline N4 C16 N3 & $126.1(9)$ \\
\hline C22 C17 C18 & $118.3(12)$ \\
\hline C17 C18 C19 & $119.0(13)$ \\
\hline C20 C19 C18 & $119.4(14)$ \\
\hline C21 C20 C19 & $122.7(16)$ \\
\hline C20 C21 C22 & $119.0(14)$ \\
\hline C17 C22 C21 & $121.6(13)$ \\
\hline C28 C23 C24 & $118.8(10)$ \\
\hline C25 C24 C23 & $121.6(11)$ \\
\hline C24 C25 C26 & $118.8(11)$ \\
\hline C27 C26 C25 & $119.2(11)$ \\
\hline C28 C27 C26 & $120.7(11)$ \\
\hline C27 C28 C23 & $120.8(10)$ \\
\hline C30 C29 C34 & $119.9(10)$ \\
\hline C29 C30 C31 & $118.9(11)$ \\
\hline C32 C31 C30 & $120.4(10)$ \\
\hline C33 C32 C31 & $119.4(10)$ \\
\hline C32 C33 C34 & $121.6(11)$ \\
\hline C33 C34 C29 & $119.7(10)$ \\
\hline N3 Ru1 N1 & $89.7(3)$ \\
\hline N3 Ru1 N1 & $89.7(3)$ \\
\hline N3 Ru1 Cl1 & $89.6(2)$ \\
\hline N3 Ru1 Cl1 & $89.6(2)$ \\
\hline N1 Ru1 Cl1 & $88.4(2)$ \\
\hline N5 P1 C29 & $110.5(3)$ \\
\hline C17 P1 C23 & $107.7(5)$ \\
\hline
\end{tabular}


TABLE 3: Continued.

\begin{tabular}{|c|c|}
\hline Atom & Angle \\
\hline C1 N1 Ru1 & $124.6(6)$ \\
\hline C9 N3 C16 & $108.0(8)$ \\
\hline C1 N4 C16 & 124.3(8) \\
\hline N4 C1 C2 & $122.3(9)$ \\
\hline C3 C2 C1 & 133.3(9) \\
\hline $\mathrm{C} 4 \mathrm{C} 3 \mathrm{H} 3$ & 121.1 \\
\hline C5 C4 H4 & 118.9 \\
\hline C4 C5 H5 & 119.4 \\
\hline C7 C6 H6 & 121.4 \\
\hline C6 C7 C8 & 132.2(9) \\
\hline N2 C8 C7 & $124.0(9)$ \\
\hline N2 C9 C10 & $122.0(9)$ \\
\hline C11 C10 C9 & $133.3(10)$ \\
\hline C12 C11 H11 & 121.3 \\
\hline C11 C12 H12 & 119.0 \\
\hline C14 C13 H13 & 119.9 \\
\hline C15 C14 H14 & 120.7 \\
\hline C14 C15 C16 & $132.3(10)$ \\
\hline N4 C16 C15 & $123.9(9)$ \\
\hline C22 C17 P1 & 119.1(9) \\
\hline C17 C18 H18 & 120.5 \\
\hline C20 C19 H19 & 120.3 \\
\hline C21 C20 H20 & 118.7 \\
\hline C20 C21 H21 & 120.5 \\
\hline C17 C22 H22 & 119.2 \\
\hline C28 C23 P1 & $122.3(8)$ \\
\hline C25 C24 H24 & 119.2 \\
\hline C24 C25 H25 & 120.6 \\
\hline C27 C26 H26 & 120.4 \\
\hline C28 C27 H27 & 119.6 \\
\hline C27 C28 H28 & 119.6 \\
\hline C30 C29 P1 & $122.1(8)$ \\
\hline С29 C30 H30 & 120.5 \\
\hline C32 C31 H31 & 119.8 \\
\hline C33 C32 H32 & 120.3 \\
\hline C32 C33 H33 & 119.2 \\
\hline C33 C34 H34 & 120.2 \\
\hline N3 Ru1 N1 & $90.3(3)$ \\
\hline N1 Ru1 N1 & $179.999(1)$ \\
\hline N1 Ru1 Cl1 & $88.4(2)$ \\
\hline N3 Ru1 Cl1 & $90.4(2)$ \\
\hline Cl1 Ru1 Cl1 & $179.999(1)$ \\
\hline C17 P1 C29 & $108.2(5)$ \\
\hline C29 P1 C23 & $108.8(5)$ \\
\hline C8 N1 Ru1 & $125.4(6)$ \\
\hline C9 N3 Ru1 & $126.1(7)$ \\
\hline P1 N5 P1 & $179.999(1)$ \\
\hline N1 C1 C2 & $108.9(8)$ \\
\hline C7 C2 C1 & $106.6(8)$ \\
\hline $\mathrm{C} 2 \mathrm{C} 3 \mathrm{H} 3$ & 121.1 \\
\hline C3 $\mathrm{C} 4 \mathrm{H} 4$ & 118.9 \\
\hline
\end{tabular}

TABle 3: Continued.

\begin{tabular}{ll}
\hline Atom & Angle \\
\hline C6 C5 H5 & 119.4 \\
C5 C6 H6 & 121.4 \\
C2 C7 C8 & $106.3(8)$ \\
N1 C8 C7 & $108.3(8)$ \\
N3 C9 C10 & $109.7(9)$ \\
C15 C10 C9 & $105.8(9)$ \\
C10 C11 H11 & 121.3 \\
C13 C12 H12 & 119.0 \\
C12 C13 H13 13 C14 H14 & 119.9 \\
C13 C10 C15 C16 & 120.7 \\
N3 C16 C15 & $106.5(8)$ \\
C18 C17 P1 & $109.9(9)$ \\
C19 C18 H18 & $122.6(9)$ \\
C18 C19 H19 & 120.5 \\
C19 C20 H20 & 120.3 \\
C22 C21 H21 & 118.7 \\
C21 C22 H22 & 120.5 \\
C24 C23 P1 & 119.2 \\
C23 C24 H24 & $119.0(8)$ \\
C26 C25 H25 & 119.2 \\
C25 C26 H26 120.6 \\
C26 C27 H27 23 H28
\end{tabular}

\section{Results and Discussion}

The low solubility of $\mathrm{Ru}^{\mathrm{III}}(\mathrm{Pc}) \mathrm{Cl}_{2}$ can be a cause of deterrent for the compound to be used as a precursor in synthesizing $\mathrm{PNP}\left[\mathrm{Ru}^{\mathrm{III}}(\mathrm{Pc}) \mathrm{Cl}_{2}\right]$ salt crystal. However, the difficulty can be overcome by a delicate mixture of $1: 1: 1: 1$ dimethylformamide: acetone: ethanol: hexane crystallization solvent which produced the title compound.

In Figure 1, it can be observed that $\operatorname{PNP}\left[\mathrm{Ru}^{\mathrm{III}}(\mathrm{Pc}) \mathrm{Cl}_{2}\right]$ units form ordered solid-state arrangement. Particularly, the anion component of the title compound, $\left[\mathrm{Ru}{ }^{\mathrm{III}}(\mathrm{Pc}) \mathrm{Cl}_{2}\right]^{-}$, affords unidirectional orientation. The crystallographic parameters of $\mathrm{PNP}\left[\mathrm{Ru}(\mathrm{Pc}) \mathrm{Cl}_{2}\right]$ are listed in Table 1 . The crystal structure of $\mathrm{PNP}\left[\mathrm{Ru}(\mathrm{Pc}) \mathrm{Cl}_{2}\right]$ is seen to be isostructural with its $\mathrm{Fe}$ homologue, $\mathrm{PNP}\left[\mathrm{Fe}^{\mathrm{III}}(\mathrm{Pc}) \mathrm{Cl}_{2}\right]$, which also has a triclinic $(Z=1)$ crystal system [3].

At the molecular level (Figure 2), the regularity is brought about by the planarity of the RuPc and the linearity of the diaxial chloro ligands which give it a uniform octahedral architecture, that is, the central $\mathrm{Ru}^{3+}$ is aligned with the planarity of the Pc moiety which is manifested by the bond lengths, as well 


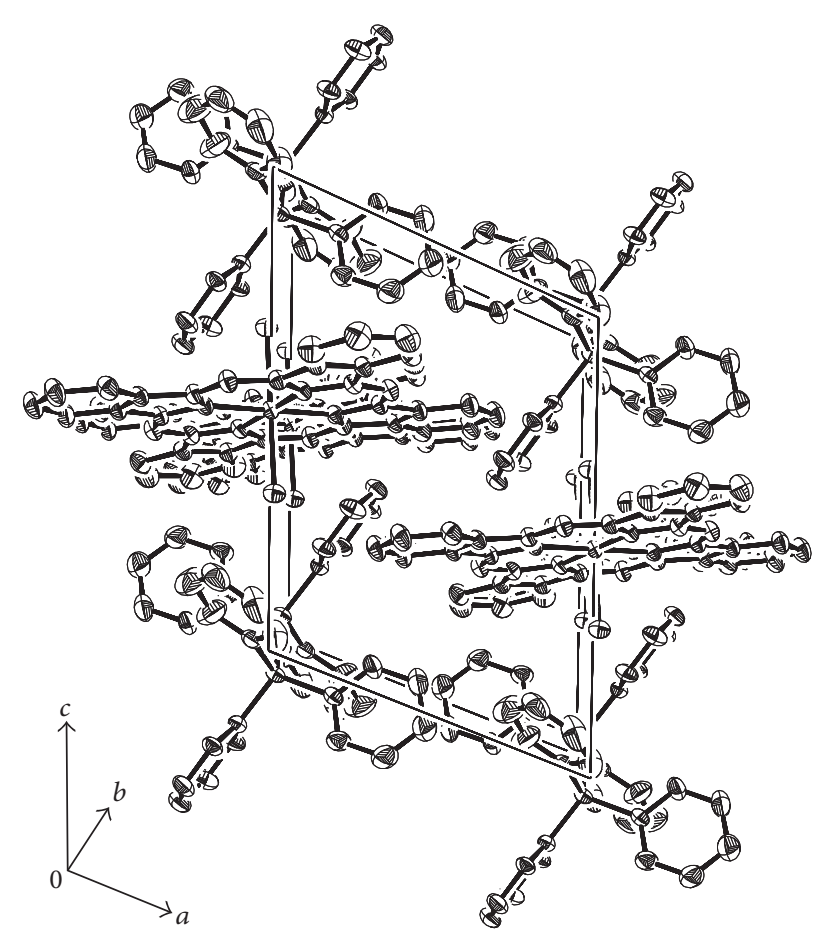

FIgURE 1: Crystal structure of $\mathrm{PNP}\left[\mathrm{Ru}^{\mathrm{III}}(\mathrm{Pc}) \mathrm{Cl}_{2}\right]$ (crystal system = triclinic; $Z=1$ ).

as the bond angles between the central $\mathrm{Ru}^{3+}$ and its adjacent nitrogen atoms being nearly equal (Tables 2 and 3). Furthermore, there is a linear $180^{\circ}$ bond angle between the two axial chloro ligands which are perpendicular $\left(90^{\circ} \pm 1.6\right)$ with respect to the central metal (Table 3), making $\left[\mathrm{Ru}^{\mathrm{III}}(\mathrm{Pc}) \mathrm{Cl}_{2}\right]^{-}$ suitable for slip-stacked intermolecular arrangement, with the cation bis(triphenylphosphine)iminium (PNP) serving as effective space-filler in the crystal system.

The resulting unidirectional and ordered orientation of $\left[\mathrm{Ru}^{\mathrm{III}}(\mathrm{Pc}) \mathrm{Cl}_{2}\right]^{-}$units is mainly attributed to the steric influence of small and linear axial ligands of the fully conjugated planar Pc from which electrical and magnetic property manifestations can be designed and modulated based on its bulkiness for corresponding intermolecular $\pi-\pi$ overlap variations [11], as well as on the chemical properties founded on the ligand field energy [3] of the axial ligands.

\section{Conclusion}

The synthesis of the crystalline $\mathrm{PNP}\left[\mathrm{Ru}{ }^{\mathrm{III}}(\mathrm{Pc}) \mathrm{Cl}_{2}\right]$ revealed an ordered octahedral structural architecture of the $\mathrm{Ru}(\mathrm{Pc}) \mathrm{Cl}_{2}$ moiety. The regularity of the structure, coupled with the steric influence of the linear axial ligands, could effectively result in a slip-stacked arrangement capable of intermolecular $\pi-\pi$ orbital overlap for electron conduction. Furthermore, $\mathrm{PNP}\left[\mathrm{Ru}^{\mathrm{III}}(\mathrm{Pc}) \mathrm{Cl}_{2}\right]$ is found to be isomorphous with its $\mathrm{Fe}$ homologue, thus opening prospects for the solid-state synthesis of other possible $\mathrm{Fe}(\mathrm{Pc}) \mathrm{L}_{2}$ homologue species of ruthenium. The resulting $\mathrm{Ru}(\mathrm{Pc}) \mathrm{L}_{2}$ is expected to have stronger $\pi$ - $d$ interactions than its $\mathrm{Fe}$ counterparts

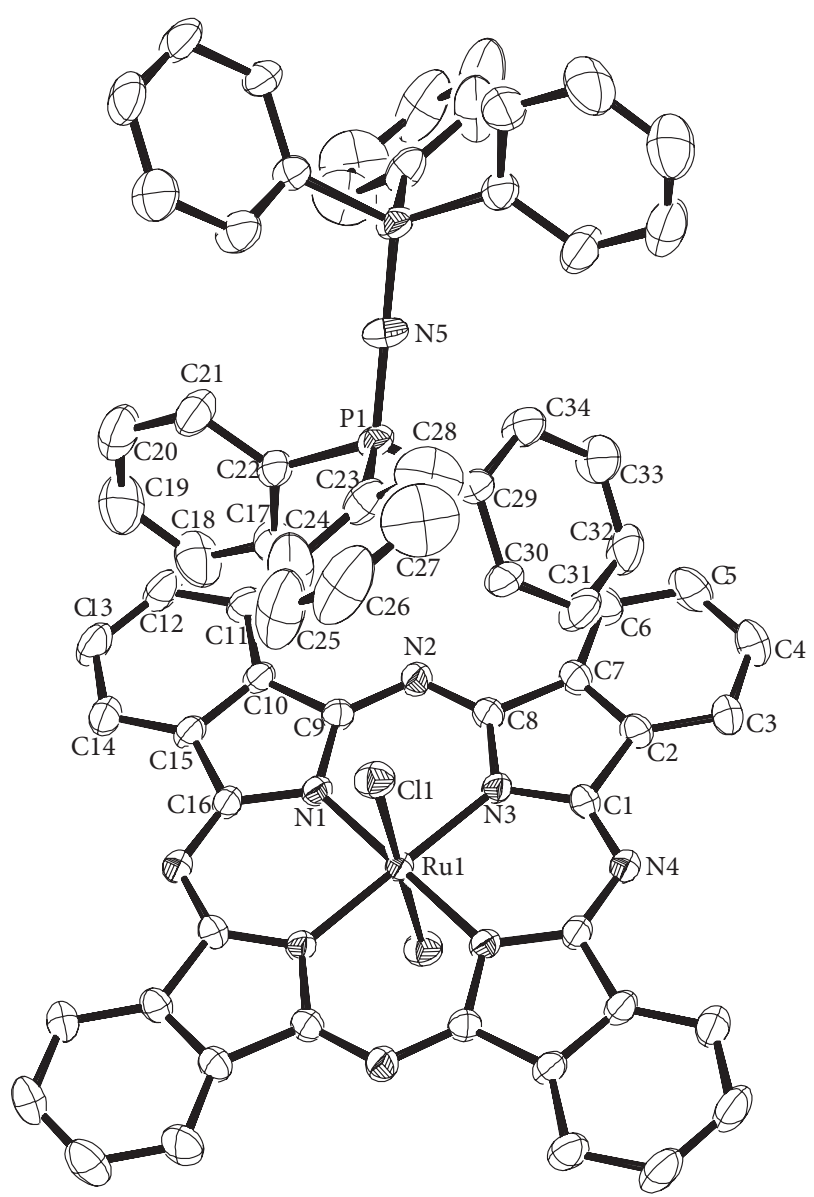

FIGURE 2: ORTEP molecular structure representation of $\mathrm{PNP}\left[\mathrm{Ru}^{\mathrm{III}}(\mathrm{Pc}) \mathrm{Cl}_{2}\right]$ (hydrogens are omitted for clarity).

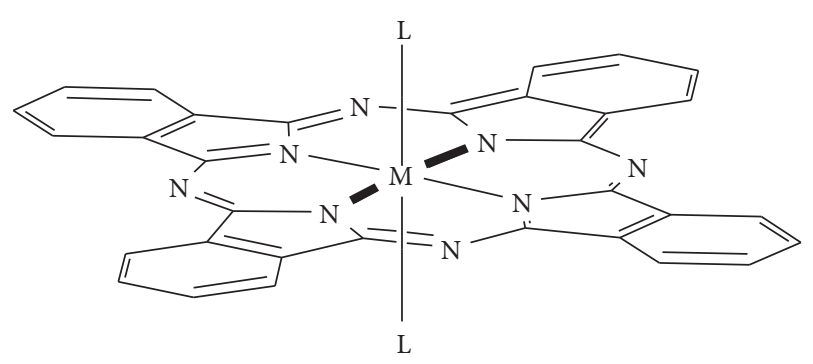

Scheme 1: Structure of $\mathrm{M}^{\mathrm{III}}(\mathrm{Pc}) \mathrm{L}_{2}$ (where $\mathrm{M}=$ central metal and $\mathrm{L}$ = axial ligands).

that could result in molecular conductors with exceptional GNMR.

\section{Appendix}

CCDC 864862 contains the supplementary crystallographic data for this paper. These data can be obtained free of charge from The Cambridge Crystallographic Data Centre via http://www.ccdc.cam.ac.uk/data_request/cif. 


\section{Acknowledgments}

This work was supported by the Hokkaido University Global Center of Excellence (GCOE) Program in chemistry and materials science (2007-2012) funded by the Ministry of Education, Culture, Sports, Science, and Technology (MEXT) of the Japan Government.

\section{References}

[1] T. Inabe and H. Tajima, "Phthalocyanines-versatile components of molecular conductors," Chemical Reviews, vol. 104, no. 11, pp. 5503-5534, 2004.

[2] D. E. C. Yu, M. Matsuda, H. Tajima, T. Naito, and T. Inabe, "Stable $\pi-\pi$ dependent electron conduction band of TPP $\left[\mathrm{M}(\mathrm{Pc}) \mathrm{L}_{2}\right]_{2}$ molecular conductors (TPP = tetraphenylphosphonium; $\mathrm{M}$ = Co, Fe; Pc = phthalocyaninato; L = CN, Cl, Br)," Dalton Transactions, vol. 40, no. 10, pp. 2283-2288, 2011.

[3] D. E. C. Yu, M. Matsuda, H. Tajima et al., "Variable magnetotransport properties in the $\operatorname{TPP}\left[\mathrm{Fe}(\mathrm{Pc}) \mathrm{L}_{2}\right]_{2}$ system $(\mathrm{TPP}=$ tetraphenylphosphonium, $\mathrm{Pc}=$ phthalocyaninato, $\mathrm{L}=\mathrm{CN}, \mathrm{Cl}$, and Br)," Journal of Materials Chemistry, vol. 19, no. 6, pp. 718-723, 2009.

[4] H. J. Werner, P. J. Knowles, R. Lindh, F. R. Manby, and M. Schutz, "2006," MOLPRO version 2006.1, a package of ab initio program, http://www.molpro.net/.

[5] L. R. Subramanian, "Tribute to Professor Dr Michael Hanack," Journal of Porphyrins and Phthalocyanines, vol. 4, no. 3, pp. 300-309, 2000.

[6] T. Rawling and A. McDonagh, "Ruthenium phthalocyanine and naphthalocyanine complexes: synthesis, properties and applications," Coordination Chemistry Reviews, vol. 251, no. 910, pp. 1128-1157, 2007.

[7] M. Weidemann, H. Hueckstaedt, and H. Homborg, "Darstellung und Eigenschaften von (Acido)(pyridin)phthalocyaninato(2-)ruthenaten(II); Kristallstruktur von Tetra(n-butyl)ammonium(cyano)(pyridin)phthalocyaninato(2-)ruthenat(II)," Zeitschrift für Anorganische Und Allgemeine Chemie, vol. 624, no. 5, pp. 846-852, 1998.

[8] J. F. Myers, G. W. Canham, and A. B. P. Lever, "Higher oxidation level phthalocyanine complexes of chromium, iron, cobalt and zinc. Phthalocyanine radical species," Inorganic Chemistry, vol. 14, no. 3, pp. 461-468, 1975.

[9] N. P. Farrell, A. J. Murray, J. R. Thornback, D. H. Dolphin, and B. R. James, "Phthalocyanine complexes of ruthenium(II)," Inorganica Chimica Acta, vol. 28, pp. L144-L146, 1978.

[10] D. J. Watkin, C. K. Prout, J. R. Carruthers, and P. W. Betteridge, "CrystalStructure 4.0: crystal structure analysis package, Rigaku and Rigaku/MSC (2010)," in CRYSTALS Issue 10, Chemical Crystallography Laboratory, Oxford, UK, 1996.

[11] D. E. C. Yu, H. Imai, M. Ushio, S. Takeda, T. Naito, and T. Inabe, "One-step synthesis of partially oxidized cobalt(III) phthalocyanine salts with axial ligands," Chemistry Letters, vol. 35, no. 6, pp. 602-603, 2006. 

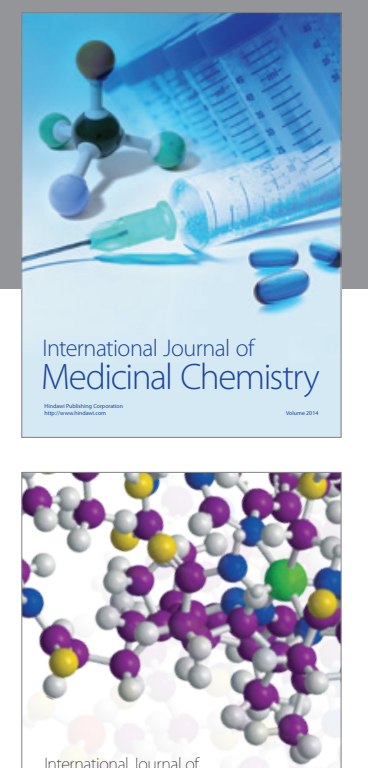

\section{Carbohydrate} Chemistry

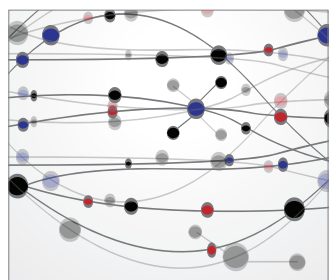

The Scientific World Journal
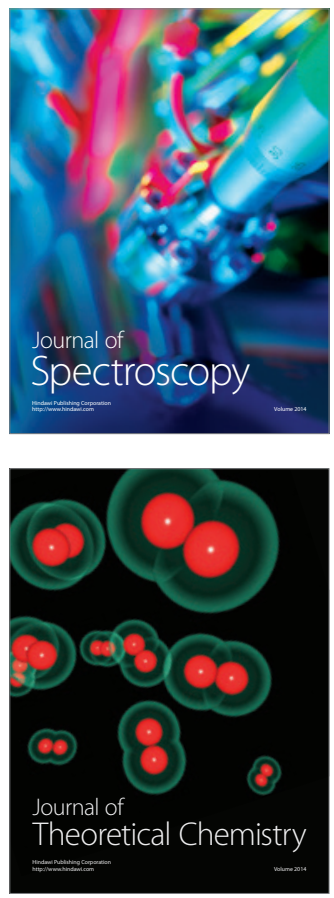
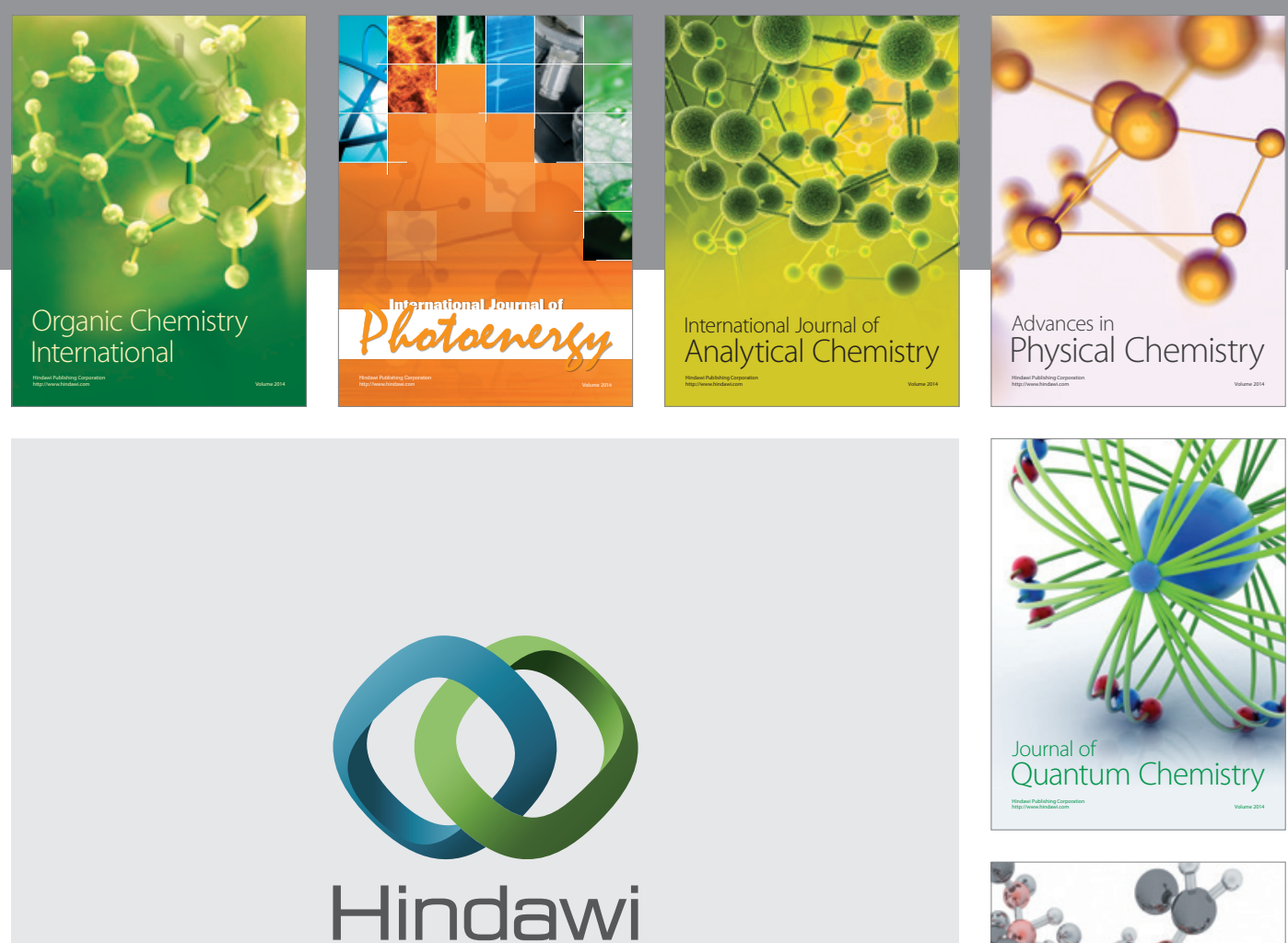

Submit your manuscripts at

http://www.hindawi.com

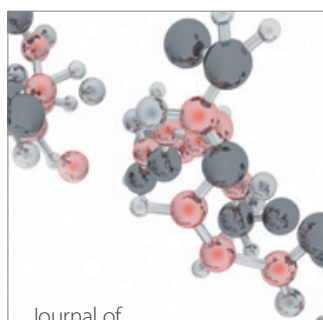

Analytical Methods

in Chemistry

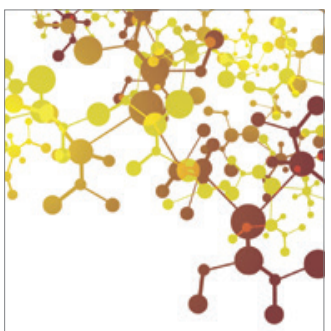

Journal of

Applied Chemistry

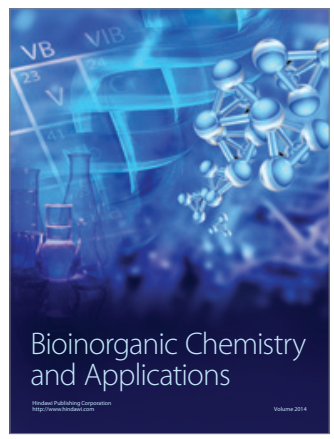

Inorganic Chemistry
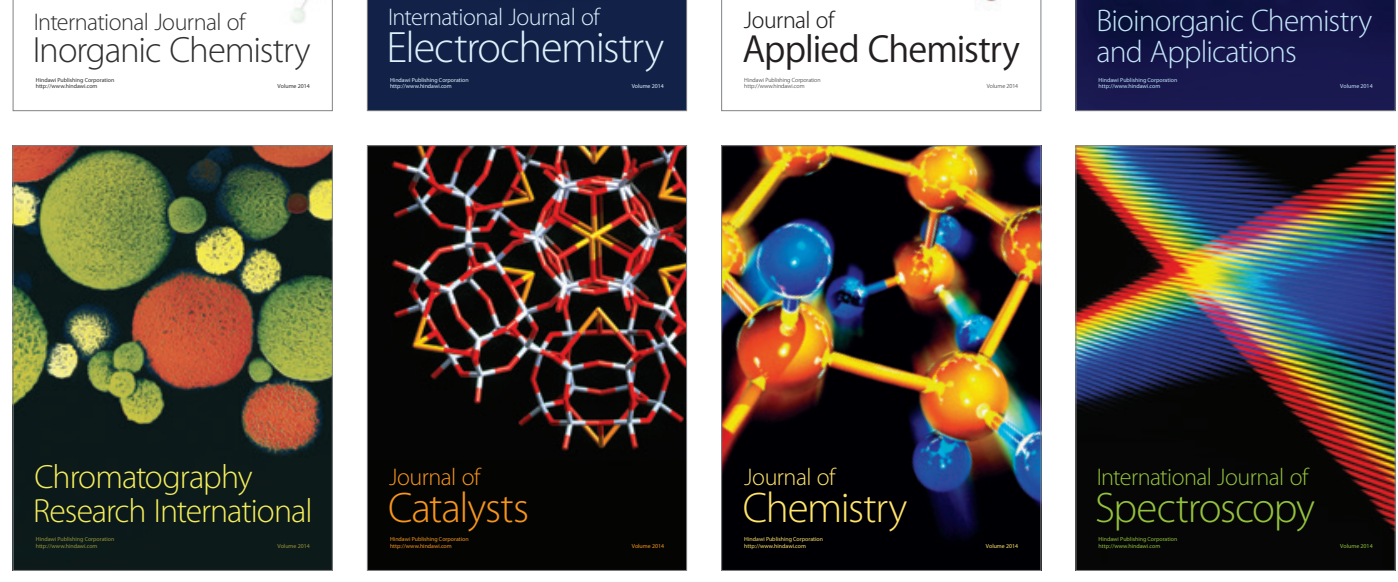